\title{
Enhanced Optical Nonlinearities in 2D-3D Heteromaterials
}

\author{
Nicolae C. Panoiu and Jian Wei You \\ Department of Electronic and Electrical Engineering, University College London, Torrington Place, London WC1E 7JE, United Kingdom
}

Remarkable optical and electrical properties of graphene [1] and other two-dimensional (2D) materials provide significant potential for novel optoelectronic applications and devices, many of which depend on nonlinear optical effects in these 2D materials [2,3]. In this paper, we will review some recent results $[4,5]$ pertaining to nonlinear optical effects in nanostructured bulk optical media containing homogeneous or nanopatterned 2D materials.

We will first introduce and briefly discuss some theoretical concepts and methods widely used in the study of optical properties of periodic structures and photonic devices, such as metamaterials, diffractive optical elements, and plasmonic structures. In particular, we will explain how the mathematical formulation of these methods can be extended to incorporate quadratic and cubic nonlinear optical effects in 2D materials, a key feature that makes these methods powerful tools for the exploration of nonlinear optical effects at the nanoscale. In the second part of the talk we will illustrate how these tools can be used to design nonlinear photonic devices with new functionalities and explore new, intriguing nonlinear optical effects at deep subwavelength scale. To this end, we will present several physical mechanisms which lead to orders-of-magnitude enhancement of optical nonlinearities in specially engineered optical structures containing 2D materials. More specifically, we demonstrate strongly enhanced nonlinear optical interactions in periodically patterned photonic nanostructures via resonant excitation of nonlinear waveguide modes, enhanced optical nonlinearity of nanostructures containing graphene and transition metal dichalcogenide nanomaterials $\left(\mathrm{MoS}_{2}, \mathrm{WSe}\right)$, and tunable Fano resonances for increasing the nonlinear conversion efficiency of hybrid 2D-3D photonic heteromaterials.

\section{References}

[1] K. S. Novoselov, A. K. Geim, S. V. Morozov, D. Jiang, Y. Zhang, S. V. Dubonos, I. V. Grigorieva, and A. A. Firsov. "Electric field effect in atomically thin carbon films," Science 306, 666-669 (2004).

[2] F. Bonaccorso, Z. Sun, T. Hasan, and A. C. Ferrari. "Graphene photonics and optoelectronics," Nat. Photon. 4, 611-622 (2010).

[3] F. Xia, T. Mueller, Y. M. Lin, A. V. Garcia, and P. Avouris, “Ultrafast graphene photodetector,” Nat. Nanotechnol. 4, 839-843 (2009).

[4] M. Weismann and N. C. Panoiu, "Theoretical and computational analysis of second- and third-harmonic generation in periodically patterned graphene and transition-metal dichalcogenide monolayers," Phys. Rev. B 94, 035435 (2016).

[5] J. W. You, J. You, M. Weismann, and N. C. Panoiu, "Double-resonant enhancement of third-harmonic generation in graphene nanostructures," Phil. Trans. R. Soc. A (to be published). 